\title{
Farmacocinética de medicamentos de uso pediátrico, visión actual
}

\author{
IVÁN SAAVEDRA S. ${ }^{1}$, LUIS QUIÑONES S. ${ }^{2}$, MÓNICA SAAVEDRA B., \\ JAIME SASSO A. ${ }^{1}$, JORGE LEÓN T. ${ }^{1}$, ANGELA ROCO A. ${ }^{2}$ \\ 1. Químico-Farmacéutico. \\ 2. Bioquímico(a). \\ Laboratorio de Farmacocinética y Biodisponibilidad, Centro de Investigaciones Farmacológicas y Toxicológicas (IFT), \\ Programa de Farmacología Molecular y Clínica, ICBM, Facultad de Medicina Universidad de Chile.
}

\begin{abstract}
Pediatric drugs pharmacokinetics

Pharmacokinetics corresponds to the branch of pharmacology that studies the absorption, distribution, biotransformation and excretion of drugs in the body, in order to proportionate a reference line for interpretation of drug concentration in biological fluids, fundamental for clinical therapy. While adult pharmacology has increase greatly, advances in pediatric pharmacology have been poor. Therefore, drug prescription in children is essentially empirical on the basis of an inmature organism. An effective, secure and rational pediatric pharmacology requires exhaustive knowledgement of the developmental changes in relation to absorption, distribution, metabolism and excretion affecting pharmacokinetics parameters; therefore, the effective dose. This review describes fundamental differences between adult and pediatric pharmacokinetics. These differences must be considered when therapeutic strategies develop for newborns and children.
\end{abstract}

(Key words: Pharmacokinetics, bioavailability, pediatric drugs).

Rev Chil Pediatr 2008; 79 (3): 249-258

\section{RESUMEN}

La farmacocinética, rama de la farmacología que estudia el paso de las drogas a través del organismo en función del tiempo y la dosis tiene por finalidad el proporcionar un marco de referencia para interpretar la concentración de los fármacos en los líquidos biológicos por el bien del paciente, lo que es fundamental para una correcta terapéutica clínica. Mientras los avances en farmacología clínica del adulto en las últimas décadas tuvieron un gran adelanto, no ha ocurrido lo mismo en farmacología pediátrica donde la mayoría de las veces la prescripción de medicamentos se realiza sobre una base empírica en un organismo

Trabajo recibido el 14 de agosto de 2007, devuelto para corregir el 02 de octubre de 2007, segunda versión el 04 de febrero de 2008, aceptado para publicación el 07 de mayo de 2008.

Correspondencia a:

Iván Saavedra S.

E-mail: isaavedr@med.uchile.cl 
inmaduro. Una terapéutica farmacológica efectiva, segura y racional en neonatos, lactantes y niños requiere el exhaustivo conocimiento de las diferencias en la absorción, distribución, metabolismo y excreción, las que aparecen durante el crecimiento y desarrollo, debido a que virtualmente, todos los parámetros farmacocinéticos se modifican con la edad. Esta revisión describe las diferencias fundamentales en la farmacocinética de los medicamentos en el niño cuando se compara con el adulto. Estas diferencias y los cambios en estos procesos deben ser cuidadosamente considerados cuando se desarrollan estrategias terapéuticas en recién nacidos y niños pequeños.

(Palabras clave: Farmacocinética, biodisponibilidad, drogas pediátricas)

Rev Chil Pediatr 2008; 79 (3): 249-258

\section{Introducción}

Durante las últimas décadas la farmacología clínica del adulto expone un gran desarrollo y avance para la mayoría de sus especialidades médicas, suceso que no se ha replicado en la farmacología pediátrica en donde muchas veces la prescripción para este grupo se realiza sobre la base empírica de un organismo inmaduro.

Cuando un fármaco penetra en el organismo ocurren secuencialmente las etapas de absorción, distribución, metabolismo o biotransformación y excreción (proceso ADME), proceso que depende de una serie de variables físicoquímicas y fisiológicas (tabla 1). Al respecto, desde el nacimiento hasta la edad adulta se producen una serie de modificaciones anatómicas, fisiológicas y bioquímicas, que afectan estos procesos farmacocinéticos, así como también algunos aspectos farmacodinámicos que afectan la acción de las drogas, la biosíntesis enzimática, la producción y distribución de receptores (figura 1). Estas modificaciones son máximas en la época perinatal con grandes cambios y necesidades adaptativas que condicionan las respuestas farmacocinéticas y de los receptores $^{1}$. El conocimiento acabado de estos cambios permite un mayor éxito en la terapia farmacológica.

Se reconoce que muchas drogas pueden variar sus efectos en lactantes y niños con respecto a los adultos, aún cuando se ha hecho cuidadosos cálculos de dosis, proporcionales al peso corporal o estimando áreas de superficie corporal $^{2}$. La tabla 2 describe las variaciones que sufren algunos parámetros farmacocinéticos desde el nacimiento al estado adulto, para diversos fármacos.

Una terapéutica farmacológica efectiva, segura y racional en neonatos, lactantes y niños requiere el conocimiento de las diferencias en el mecanismo de acción, absorción, metabolismo y excreción que aparecen durante el crecimiento y desarrollo, debido a que virtualmente, todos los parámetros farmacocinéticos se modifican con la edad ${ }^{4}$.

A continuación desarrollaremos in extenso el conocimiento actual acerca de las características farmacocinéticas de niños y sus diferencias con la respuesta del adulto.

\section{Proceso farmacocinético}

Absorción: La absorción de un fármaco y su biodisponibilidad en el organismo depende en gran medida de la vía de administración. En pediatría la vía de administración más utilizada es la oral, por lo que es necesario tener en cuenta los siguientes aspectos:

a) pH intraluminal. Después de las primeras 24 horas de vida se produce un estado de moderada aclorhidria ( $\mathrm{pH}$ de 6-7) (figura 1c), probablemente debido a ingestión de líquido amniótico y a la inmadurez del sistema de secreción ácida. El pH intraluminal alcanza los valores del adulto cerca de los 30 meses de edad $^{5}$.

b) Difusión pasiva. Depende de la liposolubilidad y el grado de ionización de los medicamentos ${ }^{6}$.

c) Vaciamiento gástrico. Es prolongado y errático, no alcanzando los valores que se dan 
Tabla 1. Factores que pueden afectar la farmacocinética de drogas

\begin{tabular}{|c|c|c|c|c|}
\hline & Absorción & $\begin{array}{l}\text { Distribución y } \\
\text { Almacenamiento }\end{array}$ & Biotransformación & Excreción \\
\hline $\begin{array}{l}\text { Factores } \\
\text { Físico- } \\
\text { Químicos }\end{array}$ & $\begin{array}{l}\text { - Peso molecular } \\
\text { - pKa y grado de ionización } \\
\text { - Coeficiente de partición } \\
\text { lípido-agua (liposolubilidad) } \\
\text { - pH y permeabilidad de la } \\
\text { membrana en el sitio de } \\
\text { - Tabsorción } \\
\text { - Tamaño de las partículas }\end{array}$ & $\begin{array}{l}\text { - Liposolubilidad } \\
\text { - Unión a proteínas } \\
\text { plasmáticas } \\
\text { - Afinidad por tejidos } \\
\text { y receptores }\end{array}$ & $\begin{array}{l}\text { - Liposolubilidad } \\
\text { - Peso molecular } \\
\text { - Isomería }\end{array}$ & $\begin{array}{l}\text { - Hidrofilicidad } \\
\text { - Tipo de metabolismo }\end{array}$ \\
\hline $\begin{array}{l}\text { Factores } \\
\text { Fisiológicos }\end{array}$ & $\begin{array}{l}\text { Gastrointestinal: } \\
\text { - pH gástrico } \\
\text { - Tiempo de vaciamiento } \\
\text { gástrico } \\
\text { - Tránsito intestinal } \\
\text { - Tipo de alimentación } \\
\text { - Superficie de absorción } \\
\text { - Microflora Gl } \\
\text { Intramuscular: } \\
\text { - Flujo sanguíneo local } \\
\text { - Masa muscular y contrac- } \\
\text { ciones musculares } \\
\text { Percutánea: } \\
\text { - Estructura de la capa córnea } \\
\text { - Hidratación cutánea }\end{array}$ & $\begin{array}{l}\text { - Flujo sanguíneo de } \\
\text { órganos y tejidos } \\
\text { - Volumen y compo- } \\
\text { sición de comparti- } \\
\text { mientos hídricos } \\
\text { - Unión a proteínas } \\
\text { - } \text { - Afinidad tisular }\end{array}$ & $\begin{array}{l}\text { - Concentración de } \\
\text { enzimas metaboli- } \\
\text { zadoras de drogas, } \\
\text { particularmente } \\
\text { hepáticas } \\
\text { - Alteraciones en el } \\
\text { flujo sanguíneo } \\
\text { hepático } \\
\text { *Ambos aspectos } \\
\text { modifican el efecto de } \\
\text { primer paso hepático }\end{array}$ & $\begin{array}{l}\text { - Flujo sanguíneo renal } \\
\text { - Filtración glomerular } \\
\text { - Secreción tubular } \\
\text { - Reabsorción tubular } \\
\text { - pH urinario }\end{array}$ \\
\hline
\end{tabular}

Tabla 2. Algunos parámetros farmacocinéticos y farmacodinámicos observados en niños en comparación con adultos

\begin{tabular}{|c|c|c|c|c|}
\hline Fármaco & $\begin{array}{l}\text { Conc. Terapéutica } \\
\text { (mg/l) }\end{array}$ & Vd $(\mathbf{l} / \mathbf{k g})$ & $\begin{array}{l}T_{1 / 2} \text { (h) (Recién Nacido } \\
\text { (Recién Nacido/Niño/Adulto) }\end{array}$ & $\begin{array}{l}\text { Unión a proteínas (\%) } \\
\text { (Recién Nacido/Niño/Adulto) }\end{array}$ \\
\hline Ampicilina & 1,5 & $0,28(\mathrm{RN} \mathrm{0,82)}$ & $2-4 / 1-1,8 / 1-2$ & $12-15 / 15-30 / 17-20$ \\
\hline Cefalotina & $0,2-10$ & $0,21-0,26$ & $1,5-2 /-/ 0,5-1$ & $72 / 90 / 65-80$ \\
\hline Cefotaxima & $16-64$ & 0,23 & RN $\uparrow / 3,4-4,6 / 0,8-1,4$ & $30-50 /-/ 33-39$ \\
\hline Nafcilina & $0,06-2$ & (RN 0,33) & $1,2-5,5 / 0,75-1,9 / 0,5-1,5$ & $69 / 89 / 70-90$ \\
\hline Cloxacilina & $0,4-6$ & 0,1 & $\mathrm{RN} \uparrow /-/ 0,5-1$ & $89 / 93 / 95$ \\
\hline Cloramfenicol & $15-25$ & $0,5-1$ & $28 / 5,94 / 1,6-3,3$ & $46 / 66 / 50-80$ \\
\hline Gentamicina & $4-8$ & $0,25(\mathrm{RN} 0,4-0,6)$ & $1,5-8,9 /-/ 1,5-4$ & $0-30 / 0-30 / 0-30$ \\
\hline Amikacina & $10-30$ & $0,27(\mathrm{RN} \uparrow)$ & $3-8 / 3-8 / 2$ & $0-10 / 0-10 / 4-11$ \\
\hline Tobramicina & $4-10$ & $0,26(\mathrm{RN} \uparrow)$ & $3,5-11,3 /-/ 1,6-3$ & $0-10 / 0-10 / 0-30$ \\
\hline Aspirina & $20-100 / 150-300 *$ & 0,15 & $-10,25-0,35 / 0,25-0,3^{* *}$ & $74 / 83 / 90$ \\
\hline Paracetamol & 10 & 1-2 (niños 0,7-1) & $2,2-5,0 / 1,0-3,5 / 2-4$ & $37 / 48 / 10-30$ \\
\hline Amoxicilina & 1,5 & $0,26-0,31$ & $3,7 / 1-2 / 0,7-1,4$ & $-/-/ 15-25$ \\
\hline Cafeína & $7-20$ & 0,54 (niños 0,85 ) & $37-231 / 5 / 4-5$ & $25 /-/ 36$ \\
\hline Teofilina & $10-20$ & $0,45(\mathrm{RN} \uparrow)$ & $12-64 / 1,9-8,5 / 6-12$ & $32-48 / 50-60 / 40$ \\
\hline Carbamazepina & $4-12$ & 1,4 & $12-64 / 1,9 / 12-17$ & $-/-176$ \\
\hline Fenitoína & $10-20$ & 0,64 & $15-105 / 2-7 / 22$ & $71-86 / 89 / 88-93$ \\
\hline Fenobarbital & $10-40$ & $0,64$ (RN $\uparrow)$ & $100-500 / 20-130 / 36-118$ & $28-43 / 48 / 20-60$ \\
\hline Ácido valproico & $50-100$ & 0,22 & $14-88 / 10 / 6-17$ & $85 / 85 / 90$ \\
\hline Diazepam & $0,1-0,25$ & 1,1 & $40-400 / 20-30 / 20-54$ & 84-98/96/94-99 \\
\hline Digoxina & $1-2,5(\mu \mathrm{g} / \mathrm{L})$ & $7(\mathrm{RN} 7,5-10)$ & $20-70 / 12-42 / 20-50$ & $14-26 / 23-40 / 22-30$ \\
\hline Furosemida & - & 0,11 (RN 0,83) & $8-27 / 0,5-3,0 / 1-3$ & 95/95/91-99 \\
\hline Warfarina & $1-3$ & 0,14 & $-/-/ 20-60$ & $-/-199$ \\
\hline Indometacina & $1,1-1,7$ & $0,34-1,57$ & $15-30 / 4,0-10 / 4,5$ & $95 / 95 / 99$ \\
\hline Propranolol & $50-100$ & 6 & $\mathrm{RN} \uparrow / 3,9-6,4 / 3-4$ & $57 / 85 / 93$ \\
\hline
\end{tabular}

* Concentración terapéutica analgésico/anti-inflamatorio

** La vida media para el ácido acetilsalićlico es de 15-20 minutos, el ácido salićlico posee un $T_{1 / 2}$ de 6 horas 
en el adulto hasta los 6 a 8 meses de edad. Durante los 2 a 4 primeros días de vida hay ausencia de peristaltismo gástrico y el estómago se vacía por una combinación de factores como el incremento del tono de la musculatura gástrica, la contracción del antro y la presión hidrostática ${ }^{7,8}$, el vaciado depende del tipo de alimentación (sólida o líquida).

d) Velocidad de absorción. Es más rápida si el fármaco está en forma líquida, siendo este tipo de formulaciones la preferida en la edad pediátrica.

e) Madurez de la mucosa intestinal, de la función biliar, de la actividad de las enzimas pancreáticas y $\beta$-glucorinidasa (figura 1c). La función biliar es inmadura en el niño y más aún en el recién nacido, asociada a una escasa secreción biliar y por consiguiente a una alteración de la absorción de drogas liposolubles.
Hasta los 4-6 meses de vida existe una deficiencia de la $\alpha$-amilasa intestinal, esto provoca una absorción irregular e incompleta en aquellos fármacos que se administran como prodrogas y que necesitan de esta enzima para hidrolizarse a su forma activa (ejemplo: cloramfenicol ${ }^{8}$ ).

f) Colonización del intestino por la flora microbiana. La flora bacteriana es capaz de metabolizar algunas drogas e influir en su biodisponibilidad, así como también en las sales biliares. Durante la vida fetal el tracto gastrointestinal es estéril. Luego del nacimiento se produce la colonización y se detectan bacterias a las 4 a 8 horas de vida. En el aparato digestivo de un recién nacido (a) de término, alimentado a pecho, predominan las especies bacilares (lactobacillus bifidus), si es a biberón es colonizado por bacterias anaeróbicas y el lactoba-
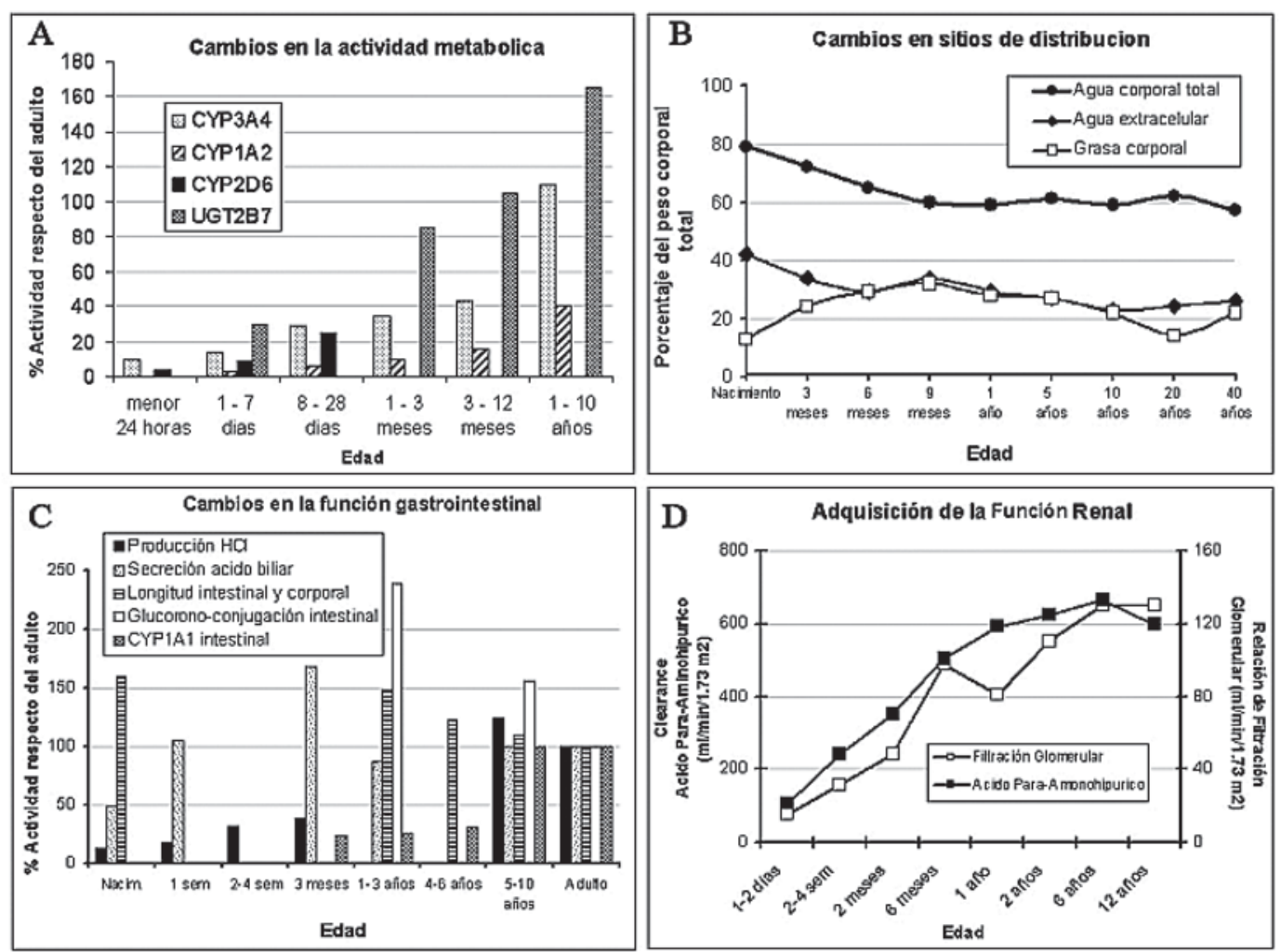

Figura 1. Cambios fisiológico-metabólicos durante el desarrollo asociados a la farmacocinética de medicamentos. Adaptado de Kearns et al., 2003. 
cillus acidophilus. Por ejemplo, es conocido que los lactantes tienen bacterias en el intestino para biotransformar la digoxina, sin embargo, la capacidad para inactivar este fármaco se desarrolla gradualmente y recién en la adolescencia se alcanzan los niveles metabólicos del adulto, lo que hace difícil establecer la dosis de este medicamento en niños ${ }^{9}$.

De estos factores, los más relevantes son, el proceso de difusión pasiva que es dependiente del $\mathrm{pH}$ y el tiempo de vaciamiento gástrico.

El peristaltismo intestinal es también irregular e impredecible y depende, en parte, del tipo de alimentación que recibe el niño. En escolares el vaciamiento gástrico y el peristaltismo son más rápidos y también hay un flujo esplácnico relativamente mayor que en el adulto lo que puede favorecer una absorción también más rápida del fármaco, con la producción de concentraciones máximas más elevadas en plasma, lo que puede dar lugar a la aparición de efectos secundarios ${ }^{7,8}$

En relación con la administración por vía rectal conviene tener en cuenta que la biodisponibilidad a que puede dar lugar es errática y no siempre se puede estar seguro de la cantidad de fármaco que va a alcanzar en el torrente sanguíneo. Por otra parte, la presencia de diarreas, dificultan la utilización de esta vía de administración. De todas formas la absorción por vía rectal en el lactante es muy buena, los datos disponibles muestran que utilizando una formulación adecuada, esta vía puede ser tan eficaz como la vía oral. Numerosos estudios indican que en terapéutica de urgencia, en estado epiléptico o en convulsiones febriles, la vía rectal permite una administración rápida y efectiva de fármacos anticonvulsivantes ${ }^{10}$.

En el recién nacido la absorción de los fármacos que se administran por vía intramuscular es variable $\mathrm{y}$, en general pobre, debido a un flujo sanguíneo relativamente bajo y a la poca masa muscular ${ }^{7}$. El dolor asociado con las inyecciones im, el riesgo de las complicaciones y la farmacocinética impredecible deja obsoleta esta vía y sólo es utilizable en casos de emergencia $^{5}$ (penicilina benzatina casi no se usa en niños).

Los vapores de líquidos volátiles y gases anestésicos pueden administrarse por vía inha- latoria. El acceso a la circulación es rápido debido a la gran superficie de absorción que ofrecen los alvéolos y la gran vascularización del sistema. En general, las drogas que se administran por vía inhalatoria cumplen un efecto local, para el tratamiento de patologías respiratorias como asma bronquial, rinitis alérgica (ej. broncodilatadores, corticoides tópicos) ${ }^{9}$, pero debe conocerse claramente el grado de absorción de cada una de ellas para reconocer posibles efectos adversos o complicaciones generadas por su efecto sistémico.

La interacción de los alimentos sobre la biodisponibilidad del fármaco es un aspecto difícil de evaluar, sobre todo en recién nacidos y lactantes, que necesitan alimentarse frecuentemente. La biodisponibilidad de fenitoína muestra un decrecimiento debido a la interacción con alimentos ${ }^{11}$. Así también la cantidad de hierro.

\section{Transporte, distribución y almacenamien-} to: Luego de que el fármaco se absorbe e ingresa a la sangre, una parte se une a proteínas y el resto circula de forma libre, esta última fracción llega al sitio de acción donde producirá el efecto farmacológico. Las drogas que son ácidos débiles en general se unen a la albúmina, ácidos grasos y a la bilirrubina. Mientras que las drogas básicas se unen a la $\alpha$-1-glucoproteína ácida y en menor proporción a las lipoproteínas.

La unión a proteínas se encuentra reducida en neonatos por dos razones: i) la concentración total de proteínas es menor, por ej. La concentración de $\alpha-1$-glucoproteína esta en un orden de 2-3 veces menor. Las proteínas plasmáticas recién alcanzan los valores adultos entre los 10 ó 12 meses de $\operatorname{edad}^{7}$ y ii) las proteínas tienen una capacidad menor de unión en neonatos.

La interacción a nivel de transporte es otra situación que se suma a una farmacocinética, ya que si se administran dos o más drogas que compiten por el sitio de unión de cierta enzima, se puede presentar un incremento de la fracción libre de aquella droga que posea una constante de afinidad menor y en consecuencia llegar a niveles tóxicos.

El volumen de distribución aparente de un fármaco (vd) aunque no es un volumen fisioló- 
gico verdadero es un parámetro farmacocinético importante que permite saber la cantidad total de fármaco que hay en el organismo en relación con su concentración sanguínea.

La cantidad de agua corporal total y agua extracelular es mayor en el recién nacido, sobre todo en el pretérmino. Luego comienza a disminuir progresivamente y así vemos que el contenido de agua extracelular es del $65 \%$ en el recién nacido pretérmino, $45 \%$ en el recién nacido a término, $33 \%$ a los 3 meses, $28 \%$ al año de vida y $20 \%$ en el adulto (figura 1 b). Esto determina un mayor vd para antibióticos del tipo aminoglicósido en niños (comparados con adultos) $)^{4}$.

A medida que disminuye el agua corporal aumenta el porcentaje de grasa corporal que en el recién nacido es del $12-15 \%$ del peso. El contenido de grasa aumenta entre los 5 y los 10 años, para luego disminuir al llegar a los 17 años. En niñas aumenta al llegar a la pubertad (13 años) y posee aproximadamente el doble de grasa corporal en comparación con los niños. Drogas que son lipofílicas como el diazepam tienen un mayor vd en niños con alta grasa corporal $^{12}$

El conocer la estimación aproximada del volumen de distribución, permite valorar cuales van a ser los compartimentos a los que va a llegar el fármaco y orienta acerca de la eficacia que se puede conseguir con la dosis utilizada.

Metabolismo (biotransformación): Los fármacos para ser eliminados del organismo deben ser transformados en compuestos más polares e hidrosolubles (metabolitos), lo que facilita su eliminación por los riñones, bilis o pulmones. La mayoría de los fármacos se metabolizan en el hígado, aunque pueden hacerlo en otros órganos tales como pulmones, riñones, suprarrenales y piel. Por ello es importante conocer el grado de maduración de la función hepática y entender el metabolismo hepático de un fármaco.

La actividad metabólica está en general reducida en el recién nacido. Concretamente las reacciones de biotransformación fase I están reducidas en el recién nacido de término y todavía más en el prematuro, no alcanzando los valores del adulto hasta los 2 a 3 años de edad.
Las reacciones de hidroxilación están muy reducidas durante los primeros meses de edad mientras que las de des-alquilación muestran un menor grado de reducción en relación a lo que sucede en el adulto. Las reacciones en fase II (conjugaciones) también están reducidas en el niño. En general, la conjugación con ácido glucurónico no alcanza los valores del adulto hasta aproximadamente los 24 a 30 meses de edad (figura 1a), por ello las drogas que necesitan este proceso tienden a acumularse en el organismo. El cloranfenicol administrado a las dosis habituales puede producir el síndrome gris, potencialmente mortal, como consecuencia de su deficiente metabolización. De las reacciones de conjugación la sulfatación es la única actividad enzimática totalmente madura al nacimiento. La concentración de ligandina o péptido y puede influir en la metabolización de fármacos en el hígado fetal. Esta proteína básica es la responsable de la captación de sustratos por las células metabolizadoras, la ligandina se une a la bilirrubina y otros compuestos (incluidos los fármacos $)^{13}$.

En general, la actividad enzimática de estos sistemas se va incrementando gradualmente hacia el primer año de vida, en forma conjunta con las proteínas plasmáticas.

Excreción: Las drogas son eliminadas del organismo en forma inalterada (moléculas de la fracción libre) o en la mayoría de los casos, como metabolitos activos o inactivos. El riñón, principal órgano excretor de fármacos inicia su maduración durante el embarazo y termina durante la primera infancia (figura 1d). Las drogas se excretan por filtración glomerular y por secreción tubular activa, siguiendo los mismos pasos y mecanismos de los productos del metabolismo intermediario. Así, las drogas que filtran por el glomérulo sufren también los procesos de reabsorción tubular pasiva. Dado que los mecanismos excretores renales no están desarrollados por completo al nacer, ello condiciona la farmacocinética de numerosas drogas ${ }^{9}$. Por otro lado, la filtración glomerular de un fármaco depende de la unión a proteínas plasmáticas (sólo la fracción libre puede filtrar), del flujo sanguíneo renal y de la superficie de filtración y dado que estos factores se modifican con la 
maduración, existen importantes diferencias interindividuales. $\mathrm{Al}$ respecto, el recién nacido de menos de 34 semanas posee menos nefrones que el recién nacido a término, la velocidad de filtración y el flujo sanguíneo renal recién alcanzan los valores del adulto a los 2 años de vida.

Las drogas que se eliminan fundamentalmente por vía renal (furosemida, aminoglucósidos, vancomicina), presentan vida media muy prolongada en el recién nacido. Se ha observado un clearence menor en la primera semana de vida, que va corrigiéndose a medida que se desarrolla la función renal, de allí la importancia de la monitorización de las concentraciones plasmáticas de aminoglucósidos sobre todo en recién nacido de pretérmino y de bajo peso, y en los gravemente enfermos, lo mismo que en niños que presentan insuficiencia renal ${ }^{9}$.

\section{Algunos ejemplos de medicamentos de uso pediátrico}

A continuación describiremos el estado del arte, en relación a estudios de farmacocinética, de algunos medicamentos de uso en pediatría.

Cisaprida: Este fármaco llegó a ser ampliamente utilizado como agente proquinético para el reflujo gastroesofágico en niños antes que se conociera de sus efectos en la arritmia cardíaca, es por ello que después de 80 casos fatales reportados, la FDA se encuentra estudiando su prohibición, por lo que se considera un medicamento en retirada, de hecho la industria farmacéutica Janssen ha suspendido la promoción de cisaprida (Prepulsid) en los EEUU a partir de julio del 2000 y continuará produciendo la droga para los pacientes que reúnan los criterios de ser elegibles para un limitado protocolo de investigación sobre sus efectos. Estudios farmacocinéticos posteriores han señalado que los niveles plasmáticos de cisaprida son dependientes del estado de desarrollo debido a la variación en la actividad de la enzima CYP3A4, responsable del metabolismo del fármaco. Para confirmar estos hallazgos Kearns et al (2003) ${ }^{14}$, reportaron un estudio con cisaprida en 35 niños de 28 a 54 semanas. Los resultados mostraron una velocidad de eliminación menor en los pacientes más jóvenes. Los autores concluyeron que la farmacocinética de cisaprida debería ajustarse tomando en cuenta esta respuesta desarrollo-dependiente de modo de reducir la toxicidad durante la infancia.

En Chile, producto de las publicaciones de la WHO, FDA y otras agencias reguladoras, se modificaron resoluciones previas que recomendaban contraindicar el uso en los pacientes de mayor riesgo y actualmente el ISP ha normado que no debe administrarse a pacientes cuya situación basal predisponga a arritmias cardíacas o en conjunto a medicamentos que prolonguen el intervalo QT o que sean inhibidores del CYP3A4 (antibióticos macrólidos, antihistamínicos, antidepresivos, antiarrítmicos, antimicóticos, inhibidores de proteasa del VIH, antipsicóticos, antinauseosos) y en resolución expresa agrega: los rótulos de los productos que contengan cisaprida cualquiera sea su forma farmacéutica y potencia deberán señalar en forma destacada la leyenda: este producto es sólo para adultos no usar en niños ${ }^{15}$.

Ketoprofeno: Un anti-inflamatorio no esteroidal útil contra el dolor. Un estudio realizado en Finlandia evaluó la farmacocinética intravenosa y rectal de ketoprofeno en 28 niños entre 7 y 93 meses de edad. La dosis intravenosa produjo picos de concentración plasmática de 10,5 a $22,2 \mathrm{mg} / 1$, mientras la dosis rectal produjo concentraciones más bajas $(3,8$ a 7,4 mg/l). La biodisponibilidad de la formulación rectal fue de $73 \%$, el volumen de distribución fue de 0,04 a $0,1 \mathrm{l} / \mathrm{kg}$ y de 0,08 a $0,16 \mathrm{l} / \mathrm{kg}$, para la dosis intravenosa y rectal, respectivamente. La vida media del fármaco fue similar en ambos tratamientos $(0,7$ a $3 \mathrm{hr}$ y 1,2 a $2,9 \mathrm{hr}$ para iv y rectal, respectivamente) ${ }^{16}$.

Omeprazol: Kearns et al, $2003^{17}$ estudiaron perfiles farmacocinéticos para omeprazol en 37 niños entre 2 y 16 años de edad después de la administración de 10 ó $20 \mathrm{mg}$. Se evaluó además la presencia funcional de CYP2C19 y sus alelos, de modo de establecer el efecto del genotipo en su metabolismo. La concentración máxima observada fue $331,1 \pm 333,6 \mathrm{ng} / \mathrm{ml}$ y la vida media fue de $2,1 \pm 1,2 \mathrm{hrs}$. No se encon- 
traron diferencias en el clearence con respecto a los alelos. Los autores concluyeron que no existía diferencias farmacocinéticas entre niños y adultos, y que el genotipo no influenciaba las concentraciones plasmáticas.

Loratadina: Este fármaco es un antihistamínico tricíclico potente, de acción prolongada, con actividad selectiva, antagónica de los receptores $\mathrm{H}_{1}$ periféricos. Un estudio realizado por Salmun et al (2000) ${ }^{18}$, investigó los parámetros farmacocinéticos, además de los efectos electrocardiográficos y la tolerabilidad de este medicamento y su metabolito (desloratadina) en 18 niños entre 2 y 5 años. Las concentraciones plasmáticas de loratadina $\mathrm{y}$ desloratadina se determinaron hasta las $72 \mathrm{hrs}$ post administración. Además se realizó un estudio de tolerabilidad en 60 niños alérgicos, en un tratamiento por 15 días. En el estudio farmacocinético se encontraron concentraciones plasmáticas máximas de 7,78 y 5,09 ng/ml para loratadina y desloratadina, respectivamente, observadas a las 1,17 y 2,33 hrs después de la administración. Las monodosis y dosis múltiples fueron bien toleradas y no presentaron eventos adversos. Los parámetros electrocardiográficos no fueron alterados y todas las evidencias clínicas apuntan a una similaridad entre la dosis de $5 \mathrm{mg}$ para estos niños y la dosis diaria de $10 \mathrm{mg}$ para adultos.

Amoxicilina-clavulanato: La combinación de amoxicilina y ácido clavulánico se usa para tratar ciertas infecciones causadas por bacterias, incluyendo infecciones en los oídos, pulmones, senos, piel y vías urinarias. Amoxicilina es un antibiótico bactericida semisintético de amplio espectro cuya absorción oral es de $75 \%$ a $90 \%$, la que no es afectada por los alimentos, y su unión a las proteínas es baja; $68 \%$ de la droga inalterada se excreta por vía renal. No se conocen enzimas humanas asociadas a su metabolismo. La amoxicilina se distribuye bien y con rapidez por todo el organismo, alcanzando altas concentraciones en músculo, hígado y riñón. Por otra parte, el ácido clavulánico pertenece a la clase de medicamentos inhibidores de betalactamasa, es estructuralmente parecido a las penicilinas y funciona evitando que las bac- terias degraden la amoxicilina mediante el bloqueo del sitio activo de éstas. Uno de los primeros estudios que evaluó las características farmacocinéticas de esta asociación medicamentosa en niños fue realizado por Schaad et al, en $1983^{19}$ en 12 niños entre 2 y 14 años. La dosis utilizada fue de $25 \mathrm{mg}$ de amoxicilina y $5 \mathrm{mg}$ de ácido clavulánico por $\mathrm{kg}$ de peso corporal. El promedio de las concentraciones plasmáticas obtenidas fueron de $89,4 \mu \mathrm{g} / \mathrm{ml}$ de amoxicilina y $19,5 \mathrm{pg} / \mathrm{ml}$ de ácido clavulánico. Las vidas medias observadas fueron de 1,2 y $0,8 \mathrm{hrs}$, respectivamente. El estudio demuestra la compatibilidad de ambos medicamentos en la formulación y que la dosis investigada es apropiada para uso en niños.

Topiramato: Ferrari et al $(2003)^{20}$, evaluaron la influencia de la dosis, la edad y la concomitancia entre anticonvulsivantes y topiramato. Se estudiaron 51 pacientes de 3 a 30 años, en los que se observó una relación lineal entre la dosis y las concentraciones plasmáticas. Las dosis normalizadas fueron correlacionadas positivamente con la edad. Contrariamente, el clearance fue inversamente relacionado a la edad y directamente relacionado al uso de inductores.

Zidovudina: Aunque el perfil farmacocinético de este fármaco ha sido estudiado en varias investigaciones, muy pocos niños han sido incluidos. Como parte de un estudio pediátrico multicéntrico se evaluaron los parámetros farmacocinéticos de zidovudina, didanosina y la combinación de ellos (Caparelli et al, 2003) ${ }^{21}$. La variable más significativa que afecta su farmacocinética fue la edad. Lo sujetos de menos de dos años de edad poseen un clearence reducido. Los autores sugieren que esta característica conduce a mayor riesgo en niños debido a altas concentraciones plasmáticas de zidovudina.

Paracetamol: Este medicamento (acetaminofen) tiene como principal ventaja que puede ser administrado vía oral, endovenosa o rectal, aunque la vía rectal es lenta y de absorción irregular. Además puede ser usado en embarazo y lactancia. Ha sido demostrado que posee 
una farmacocinética lineal, es decir, que es independiente de la dosis y constante con administraciones repetidas. Paracetamol posee muy bajo nivel de unión a proteínas plasmáticas y un metabolismo mediado principalmente por conjugación lo que hace que tenga bajas posibilidades de interacción con otras drogas. La edad influencia la farmacocinética, razón por la cual resulta particularmente difícil definir la dosis ideal en niños, sobre todo para la vía rectal. Sin embargo, una dosis oral de $15 \mathrm{mg} / \mathrm{kg}$ cada $4 \mathrm{hrs}$ $\mathrm{y}$ hasta un total de $60 \mathrm{mg} / \mathrm{kg} / \mathrm{día}$ es usualmente suficiente para alcanzar el efecto analgésico y antipirético deseado ${ }^{22}$.

Un estudio realizado por Anderson et al $(2000)^{23}$, describió la farmacocinética de paracetamol en neonatos e infantes $(n=30)$ y la compararon con datos de 4 estudios similares ( $\mathrm{n}=221$ ) para dosis entre 30 a $40 \mathrm{mg} / \mathrm{kg}$ durante 2 días y concluyeron que el clearence de paracetamol al nacer es de $62 \%$ y el volumen de distribución es $174 \%$ comparado con niños mayores. Concluyeron además que una concentración de aproximadamente $10 \mathrm{mg} / \mathrm{l}$ puede ser alcanzada con una dosis de $45 \mathrm{mg} /$ $\mathrm{kg} /$ día al nacer y con una dosis de $90 \mathrm{mg} / \mathrm{kg} /$ día en niños de 5 años de edad.

\section{Discusión}

La investigación clínica en pacientes pediátricos actualmente es impulsada tanto por la FDA como por la industria farmacéutica. Aunque varios estudios han sido publicados en los últimos años, la investigación en este campo aún es muy deficiente, particularmente en nuestro país, sin embargo, es indudable que la identificación de dosis óptimas de fármacos en niños resulta prioritaria.

La intensificación de la acción de las drogas o la aparición de toxicidad en niños, reflejan diferencias con el adulto, tanto farmacocinéticas como farmacodinámicas. Conociendo estas diferencias, se debe tener especial precaución cuando se prescriben fármacos a niños enfermos, sobre todo en tratamientos prolongados ya que pueden afectar los procesos de desarrollo y crecimiento y generar importantes efectos adversos, que a veces no ocurren en el adulto.
Considerar al niño, en especial al recién nacido, como si fuera un adulto pequeño, ha producido casos de severa iatrogenia medicamentosa: kernicterus (sulfas), síndrome gris (cloranfenicol), sorderas (aminoglucósidos), etc. Por otro lado, la mayoría de los fármacos pueden atravesar la placenta y actuar sobre un ser en desarrollo, provocando malformaciones estructurales (talidomida, antineoplásicos, antiepilépticos, etc) retraso en el crecimiento intrauterino (cocaína, anfetamina, nicotina, etc), teratogenicidad (benzodiazepinas, tranquilizantes mayores, etc) o dificultades en la adaptación funcional neonatal (opiáceos y otros depresores) ${ }^{9}$.

La administración de fármacos en pediatría presenta problemas, no solamente por las diferencias de biodisponibilidad en las distintas edades, sino porque la droga puede afectar por sí misma los procesos de crecimiento y desarrollo. El efecto causado puede expresarse muchos años después de la administración de la droga (corticoides, hormonas sexuales, hormonas tiroideas, drogas antitiroídeas, etc).

En resumen, existen considerables diferencias en la farmacocinética de los medicamentos en el niño cuando se compara con el adulto. Estas diferencias y los cambios en estos procesos deben ser cuidadosamente considerados cuando se desarrollan estrategias terapéuticas en recién nacidos y niños pequeños.

Al prescribir un medicamento, el médico debe considerar las variables farmacocinéticas y farmacodinámicas. Para ello es deseable la confluencia y comunicación bilateral de conocimientos entre el profesional químico-farmacéutico y los profesionales clínicos como partes del equipo de salud

Las complejidades del cuidado farmacológico en niños son multifactoriales pero no inabordables. Los profesionales de la salud debemos trabajar juntos para utilizar en forma segura y ventajosa los fármacos en el paciente pediátrico.

\section{Referencias}

1.- Behrman R, et al: Tratado de Pediatría. 17 a edición, McGraw hill interamericana, Madrid, 2004.

2.- Avery: Neonatología, 3a. Edición, ed. Panamericana, Canadá, 1990.

3.- Heusser F, Garay F: Fármacos de uso pediátrico, QT 
prolongado y trastornos del ritmo cardíaco. Rev Chil Pediatr 1999; 70 (6): 457-63.

4.- Crom WR: Pharmacokinetcis in child. Environ Health Perspect 1994; 102 (supl 11): 111-18.

5.- Bartelink IK, Rademaker CMA, Schobben AFAM, Van Den Ander JN: Guidelines on pediatric dosing on the basis of developmental physiology and pharmacokinetic considerations. Clin Pharmacokinet 2006; 45 (11): 1077-97.

6.- Goodman \& Gilman: Laurence Brunton, undécima edición, McGraw-Hill Interamericana, México, DF, 2006.

7.- Strolin Benedetti M, Baltes EL: Drug metabolism and disposition in children. Fundam Clin Pharmacol 2003; 17: 281-99

8.- Kearns GL: Impact of developmental pharmacology on pediatric study desing; overcoming the challenges. J Allergy Clin Immunol 2000; 106: s128-39.

9.- Malgor LA, Valsecia ME: Farmacología médica, volumen 4, cap. 4: farmacocinética y farmacodinamia en pediatría, 2000. Base de datos: cátedra farmacología, Facultad de Medicina, Universidad Nacional del Nordeste, República Argentina.

10.- Ogutu BR, Newton CR, Crawley J, et al: Pharmacokinetics and anticonvulsant effects of diazepam in children with severe falciparum malaria and convulsions. Br J Clin Pharmacol 2002; 53 (1): 49-57.

11.- Albani $M$, Wernicke I: Oral phenytoin in infancy: dose requirement, absorption, and elimination. Pediatr Pharmacol (New York) 1983; 3 (3-4): 229-36.

12.- Mislap RL, Hill MR, Szefler SJ: Special pharmacokinetic considerations in children: in applied pharmacokinetics: principles of therapeutic drug monitoring, 3er. Ed. Vancouver Whasington: applied therapeutics, 1992; 10.1-10-32

13.- Matsubara T, Yamada N, Mitomi T, et al: Cytochrome p-450-dependent monooxygenase activities in prenatal and postnatal human livers: comparison of human liver 7-alkoxycoumarin o-dealkylases with rat liver enzymes. Jpn J Pharmacol 1986; 40 (3): 389-98.
14.- Kearns GL, Robinson PK, Wilson JT, et al: Cisapride disposition in neonates and infants: in vivo reflection of cytochrome p450 3a4 ontogeny. Clin Pharmacol Ther 2003; 74: 312-25.

15.- Cenimef centro nacional de información de medicamentos y farmacovigilancia. Instituto de Salud Pública de Ce, www.ispch.cl resolución exenta $\mathrm{n}^{\circ} 2586,2002$, resol.ución exenta $\mathrm{n}^{\circ} 3668,2002$, resolución exenta $\mathrm{n}^{\circ}$ 4392, 2001.

16.- Kokki H, Karvinen M, Suhonen P: Pharmacokinetics of intravenous and rectal ketoprofen in young children. Clin Pharmacokinet 2003; 42: 373-9.

17.- Kearns GL, Andersson T, James LP, et al: Omeprazole disposition in children following single-dose administration. J Clin Pharmacol 2003; 43: 840-8.

18.- Salmun LM, Herron JM, Banfield C, Padhi D, Lorber $R$, Affrime $M B$ : The pharmacokinetics, electrocardiographic effects, and tolerability of loratadine syrup in children aged 2 to 5 years. Clin Ther 2000; 22 (5): 613-21.

19.- Schaad UB, Casey PA, Cooper DL: Single-dose pharmacokinetics of intravenous clavulanic acid with amoxicillin in pediatric patients. Antimicrob Agents Chemother 1983; 23 (2): 252-5.

20.- Capparelli EV, Englund JA, Connor JD, et al: Population pharmacokinetics and pharmacodynamics of zidovudine in hiv-infected infants and children. J Clin Pharmacol 2003; 43: 133-40.

21.- Kearns GL, Abdel-Rahman SM, Alander, et al: Developmental pharmacology - drug disposition, action, and therapy in infants and children. $\mathrm{N}$ Engl J Med 2003; 349: 1157-67.

22.- Bannwarth B, Péhourcq F: Pharmacologic basis for using paracetamol: pharmacokinetic and pharmacodynamic issues. Drugs 2003; 63 (2): 5-13.

23.- Anderson BJ, Woollard GA, Holford NH: A model for size and age changes in the pharmacokinetics of paracetamol in neonates, infants and children. $\mathrm{Br} \mathrm{J}$ Clin Pharmacol 2000; 50 (2): 125-34. 